\title{
Spontaneous Spinal Epidural Hematoma During Simultaneous Tocilizumab and Warfarin Use in a Patient With Rheumatoid Arthritis: Is There a Drug Interaction Between Tocilizumab and Oral Anticoagulants?
}

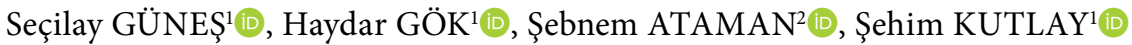 \\ ${ }^{1}$ Department of Physical Medicine and Rehabilitation, Ankara University Faculty of Medicine, Ankara, Turkey \\ ${ }^{2}$ Department of Physical Medicine and Rehabilitation, Division of Rheumatology, Ankara University Faculty of Medicine, Ankara, Turkey
}

\begin{abstract}
Interleukin 6 (IL-6) plays a main role in the immunopathogenesis of rheumatoid arthritis (RA). Tocilizumab (TCZ) is a humanized immunoglobulin G1 monoclonal antibody against the human IL-6. Warfarin sodium is an oral anticoagulant that is primarily metabolized by cytochrome P450 2 C9 (CYP2C9). Impaired metabolism of this low therapeutic index drug is important as it may result in serious bleeding. In this article, we present a 56-year-old female patient with RA, treated with TCZ and warfarin sodium and presented spontaneous spinal epidural hematoma (SSEH) of thoracic spine although international normalized ratio levels were in normal ranges. One week after decompressive surgery for hematoma, a cervical spine abscess developed which resulted in her death. To the best of our knowledge, this is the first case of RA developing SSEH while taking TCZ and warfarin sodium together. Although it is difficult to attribute the severe bleeding to TCZ treatment, clinicians should be aware that concomitant use of oral anticoagulants and TCZ might result in potentially fatal complications in patients with RA.

Keywords: Oral anticoagulant, rheumatoid arthritis, spinal epidural hematoma, tocilizumab.
\end{abstract}

Spontaneous spinal epidural hematoma (SSEH) is defined as blood within the epidural space without any known traumatic or iatrogenic cause and incidence of SSEH is 1:100,000/year. ${ }^{1}$ $\mathrm{SSEH}$ is considered as a neurological emergency and many authors suggest a relationship between SSEH and arteriovenous malformations, anticoagulant use, underlying coagulopathy and vertebral hemangiomas. ${ }^{2}$ Warfarin is the most commonly used oral anticoagulant, occurs as a pair of enantiomers that are differentially metabolized by human cytochromes P450 (CYP). ${ }^{3}$ Tocilizumab (TCZ), which is a new drug targeting the interleukin 6 (IL-6) pathway, was approved by the Food and Drug Administration for the treatment of rheumatoid arthritis (RA). Some drug interaction studies have been reported on TCZ. ${ }^{4}$ It has been highlighted that when starting or stopping therapy with TCZ, patients taking drugs metabolized via CYP450 3A4, 1A2 or 2C9 (atorvastatin, calcium channel blockers, theophylline, warfarin, phenytoin) should be monitored closely. ${ }^{3,4}$ Although reduced simvastatin and omeprazole bioavailability has been shown under TCZ treatment, the bioavailability of warfarin is not clear in the literature. ${ }^{4}$

Received: April 30, 2019 Accepted: August 17, 2019 Published online: February 07, 2020

Correspondence: Seçilay Güneş, MD. Ankara Üniversitesi Tıp Fakültesi, Fiziksel Tıp ve Rehabilitasyon Anabilim Dalı, 06590 Çankaya, Ankara, Türkiye. Tel: +90 312 - 5082822 e-mail: secilaygunes@ankara.edu.tr

\section{Citation:}

Güneş S, Gök H, Ataman Ş, Kutlay Ş. Spontaneous Spinal Epidural Hematoma During Simultaneous Tocilizumab and Warfarin Use in a Patient With Rheumatoid Arthritis: Is There a Drug Interaction Between Tocilizumab and Oral Anticoagulants? Arch Rheumatol 2020;35(4):614-617. 
In this article, we present a case of RA under TCZ and warfarin sodium therapy, presenting $\mathrm{SSEH}$ of the thoracic spine while international normalized ratio (INR) levels were in normal ranges.

\section{CASE REPORT}

A 56-year-old female patient with RA for the last 18 years had received methotrexate, methylprednisolone, leflunomide, etanercept, infliximab, and adalimumab in the past but all the drugs were discontinued due to inadequate response. TCZ therapy was started with an $8 \mathrm{mg} / \mathrm{kg} / \mathrm{month}$ infusion. She had also been using warfarin sodium due to grade 2 aortic insufficiency and mitral valve prosthesis for five years. The patient did not use any other drugs. After the $20^{\text {th }}$ day of the fourth trial of TCZ infusion, the patient was admitted to the emergency department with sudden back pain, weakness, and numbness in her legs, bladder and bowel dysfunction. There was no history of trauma. Her blood pressure was 130/90 mmHg. Neurological examination showed the presence of complete paraplegia. Laboratory findings were as follows: hemoglobin: $12.2 \mathrm{~g} / \mathrm{dL}$ (normal), platelet: $193 \times 10^{9} / \mathrm{L}$ (normal), white blood count: $6 \times 10^{9} / \mathrm{L}$ (normal), aspartate aminotransferase: $55 \mathrm{U} / \mathrm{L}$ (high), alanine aminotransferase: $50 \mathrm{U} / \mathrm{L}$ (high), blood urea nitrogen: $16 \mathrm{mg} / \mathrm{dL}$ (normal), creatinine: $1.1 \mathrm{mg} / \mathrm{dL}$ (normal), INR: 2.1 (normal), C-reactive protein (CRP): $1.2 \mathrm{mg} / \mathrm{L}$ (normal). Peripheral blood smear was normal. Factor 13 and fibrinogen levels were normal too. Urgent magnetic resonance imaging (MRI) of the thoracolumbar spine demonstrated an elongated space-occupying mass with convexity to the spinal canal at the level of T8-T12 vertebra which was seen as a hyperintense mass on the T2 weighted images and hypointense on the $\mathrm{T} 1$ weighted images (Figure 1). It was observed via immediate exploratory surgery that there was blood in the epidural space and decompressive laminectomy was performed. A written informed consent was obtained from the relatives of patient.

Seven days after the surgery, her symptoms worsened and she also developed arm weakness. Cervical MRI revealed an intramedullary mass between the C5-C7 vertebra levels which was consistent with an abscess (Figure 2) although the CRP levels were within the normal range. She was re-operated and the intradural abscess was drained. The culture of pus from the abscess turned out to be negative. She developed severe pneumonia. The blood culture was negative. One month after the first surgery, Candida albicans was observed in the culture of sputum in the intensive care unit and patient died despite the antifungal treatment.

\section{DISCUSSION}

Spontaneous spinal epidural hematoma is an extremely rare entity. RA, Paget's disease and
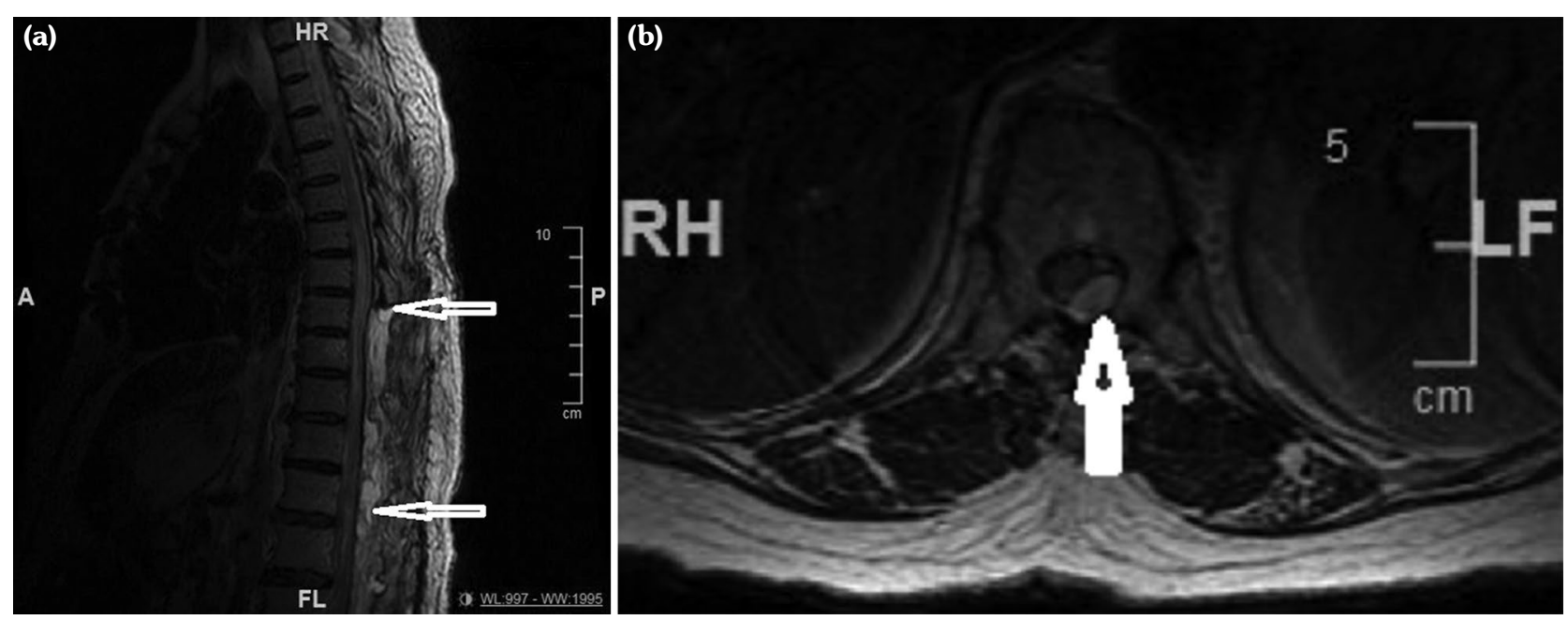

Figure 1. (a) Sagittal T2 weighted images: Hyperintense lesion between T8-T12 vertebra levels. (b) Axial T2 weighted images: Hematoma compressing spinal cord. 


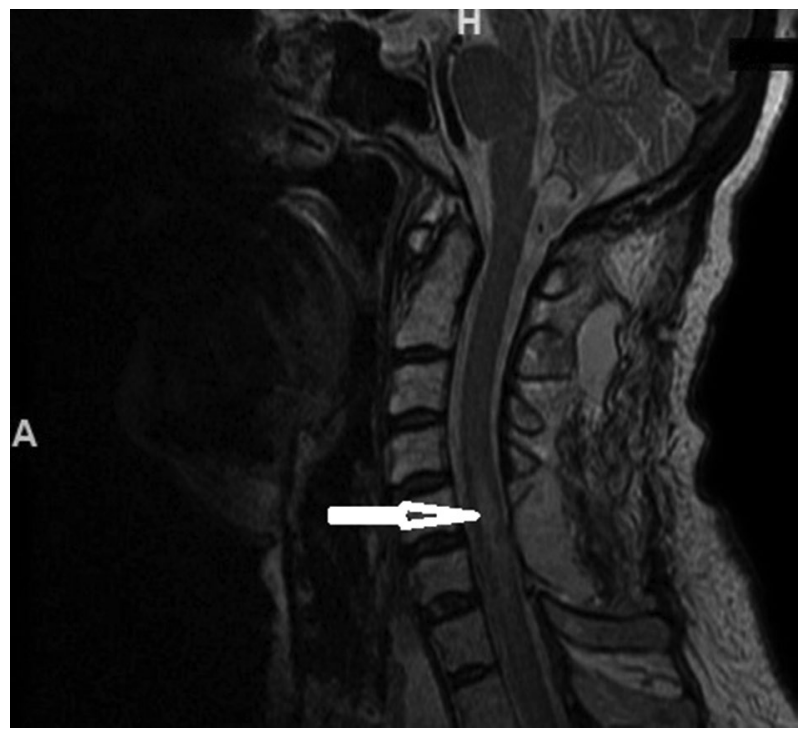

Figure 2. T2 weighted images: Cervical abscess.

ankylosing spondylitis are considered as risk factors for traumatic spinal epidural hematomas due to increased risk for compression fractures. ${ }^{5}$ There was no history of trauma in our patient with RA. However, it is known that longstanding RA patients have increased risk for vasculopathy. ${ }^{6}$ In addition, oral anticoagulation use is a risk factor for SSEH while the pathology is not clear. Some have argued that the risk of oral anticoagulation associated hemorrhage is not related to the intensity of anticoagulation. ${ }^{7}$ There is no absolutely "safe" INR, and it is noteworthy that many of the reported cases were anticoagulated in the therapeutic range. ${ }^{7}$ Therefore, any drug interactions altering the bioavailability of oral anticoagulants are important. TCZ inhibits the downregulation of the CYP2C9 which has a major role in warfarin metabolism. Aitken et al. ${ }^{8}$ showed that IL-6 induced diminution of CYP2C9 protein level which was greater than that of CYP3A4 in vitro. This suggests that CYP2C9 activity may increase at greater extent than CYP3A4 with TCZ treatment, resulting in increased warfarin metabolism. The authors stated that clinically, this would result in patients requiring a smaller dose of warfarin and a possible need for more frequent INR monitoring.

Another risk of bleeding is thrombocytopenia due to TCZ treatment. One serious bleeding case with hemorrhagic stomatitis was reported. ${ }^{9}$
Mokuda et al. $^{10}$ reported hematoma in a patient with hip pain and found a relationship between TCZ treatment and factor XIII deficiency and decreased fibrinogen. They reported that RA patients who are treated with $\mathrm{TCZ}$ are at risk for developing acquired factor XIII deficiency. However, the factor XIII level was normal in the current case.

Inhibiting IL- 6 can also be an additional factor for bleeding, by decreasing plasma fibrinogen level in patients treated with TCZ which was within the normal range as it was in our case. Imamura et al. ${ }^{11}$ showed that TCZ treatment is associated with reduced fibrinogen levels and increased blood loss after total knee arthroplasty in patients with RA.

Spontaneous spinal epidural hematoma is a very rare and fatal condition. To the best of our knowledge, this is the first case of RA developing SSEH while taking TCZ and warfarin sodium together. Since the coagulation parameters were in the normal range, it is difficult to attribute the severe bleeding directly to the TCZ treatment. While the use of anticoagulants is a risk factor for SSEH, administering drugs affecting CYP metabolism causes additional risk for spontaneous bleeding. Although the effect of TCZ on coagulation parameters and CYP enzymes is still not well known, TCZ treatment must be administered carefully in the patients who have concomitant use of warfarin sodium even if the INR levels are in normal ranges.

\section{Declaration of conflicting interests}

The authors declared no conflicts of interest with respect to the authorship and/or publication of this article.

\section{Funding}

The authors received no financial support for the research and/or authorship of this article.

\section{REFERENCES}

1. Bhat KJ, Kapoor S, Watali YZ, Sharma JR. Spontaneous epidural hematoma of spine associated with clopidogrel: A case study and review of the literature. Asian J Neurosurg 2015;10:54.

2. Zhong W, Chen H, You C, Li J, Liu Y, Huang S. Spontaneous spinal epidural hematoma. J Clin Neurosci 2011;18:1490-4. 
3. Kaminsky LS, Zhang ZY. Human P450 metabolism of warfarin. Pharmacol Ther 1997;73:67-74.

4. Kim S, Östör AJ, Nisar MK. Interleukin-6 and cytochrome-P450, reason for concern? Rheumatol Int 2012;32:2601-4.

5. Yalbuzdağ ŞA, Erol AM, Çelik C, Solum S. Ankylosing spondylitis diagnosed after epidural hematoma and paraplegia: A case report. Arch Rheumatol 2014;29:309-13.

6. Radic M, Martinovic Kaliterna D, Radic J. Overview of vasculitis and vasculopathy in rheumatoid arthritis--something to think about. Clin Rheumatol 2013;32:937-42.

7. Hart RG, Boop BS, Anderson DC. Oral anticoagulants and intracranial hemorrhage. Facts and hypotheses. Stroke 1995;26:1471-7.

8. Aitken AE, Morgan ET. Gene-specific effects of inflammatory cytokines on cytochrome P450 2C, 2B6 and 3A4 mRNA levels in human hepatocytes. Drug Metab Dispos 2007;35:1687-93.

9. Schiff MH, Kremer JM, Jahreis A, Vernon E, Isaacs JD, van Vollenhoven RF. Integrated safety in tocilizumab clinical trials. Arthritis Res Ther 2011;13:R141.

10. Mokuda S, Murata Y, Sawada N, Matoba K, Yamada $\mathrm{A}$, Onishi $\mathrm{M}$, et al. Tocilizumab induced acquired factor XIII deficiency in patients with rheumatoid arthritis. PLoS One 2013;8:e69944.

11. Imamura H, Momohara S, Yano K, Sakuma Y, Nakayama M, Tobimatsu $H$, et al. Tocilizumab treatment in patients with rheumatoid arthritis is associated with reduced fibrinogen levels and increased blood loss after total knee arthroplasty. Mod Rheumatol 2018;28:976-80. 